\title{
Current Injection Vortex Promoter for Heat Transfer Enhancement in a Magnetohydrodynamic Duct Flow
}

\author{
Ahmad H. A. Hamid, Wisam K. Hussam, Gregory J. Sheard
}

\begin{abstract}
The quest for cleaner, cheaper and reliable energy has motivated the development of magnetic confinement fusion reactor technology as a possible means of harnessing the energy produced by nuclear fusion for power generation. However, the magnetohydrodynamic effects act to reduce the thermal-hydraulic performance of the duct flows within the cooling blankets. The present paper reports the investigation of the duct flow with current injection as a vortex promoter for the enhancement of the convection heat transfer. The results indicate a maximum enhancement of approximately $70 \%$ over the duct flow without current injection for the highest current amplitude investigated. The competing effects of the Lorentz force and Hartmann damping result in a non-monotonic trend of Nusselt number with respect to friction parameter, while increasing Reynolds number results in a monotonic increase of Nusselt number.
\end{abstract}

Keywords-MHD, quasi-2-d, heat transfer, vortex promoter

\section{Introduction}

Magnetohydrodynamics (MHD) covers phenomena in electrically conducting fluids that interact with magnetic fields. The coupled effects of the dynamics of electrically conducting fluids and electromagnetism see MHD flow problems deserving specific attention quite apart from their hydrodynamic (HD) counterparts. Broadly speaking, the fluid-magnetic field interaction occurs either naturally or in a controlled environment. An example of the latter case is the interaction that occurs in a magnetic confinement fusion reactor, where the plasma is confined within a vacuum chamber by a series of magnetic fields due to its very high temperature (which can reach values of the order of $10^{8} \mathrm{~K}$ [1]). Embedded in the protective blanket surrounding the chamber will be tritium breeder modules.

The interaction between the flow and the applied magnetic field induces an electric current. The induced current then interacts with the applied magnetic field, which results in an electromagnetic Lorentz force. This force has been shown to cause a degradation in the thermal-hydraulic performance of flows within the blanket modules [2].

Ahmad H. A. Hamid

Faculty of Mechanical Engineering, Universiti Teknologi MARA, 40450 Selangor, Malaysia

Wisam K. Hussam

Gregory J. Sheard

Department of Mechanical and Aerospace Engineering, Monash University, Victoria 3800, Australia
The cooling process in an MHD duct can be assisted by mixing of the flow via turbulence or vortical structures. Several methods have been proposed to improve the convective heat transfer, but generally the mechanism is the same: either by promoting turbulence or by generating vortical velocity fields in the flow. The vortex motion induces a significant velocity component in the transverse direction, thus improving fluid mixing in this direction and reducing thermal boundary layer thickness [3].

Suggested methods to generate these vortices include placement of an obstacle in the duct such as a cylinder or grid bars [3-6]. The inherent fundamental physics of MHD flow that result in the inhibition of time dependent bluff body wakes leads to the quest for an alternative vorticity generation mechanism that avoids the insertion of a physical body, such as the use of inhomogeneous wall conductivity [7], imposing a localised zone of magnetic field, known as magnetic obstacle [8] or by spatially varying the magnetic field [9].

A promising alternative method is injection of an electrical current through the duct wall [7]. This method allows the resulting flow to be precisely controlled via the injected current parameters [10]; indeed these parameters could even be actively controlled according to feedback from the flow conditions. The current injection method has been used in various studies of confined MHD flows, in the absence of a through-flow, though a similar attention for heat transfer augmentation in a duct flow configuration is rather scarce, despite its potential capability. The present work investigates the heat transfer enhancement in an MHD duct flows with a current injection vortex generation. We are particularly interested in a flow with Reynolds number $R e=$ 1500 and 3000 in a duct with a blockage ratio $\beta=0.2$. Owing to the fact that there is a limited number of studies on an electrically driven vortices in an MHD duct flow in the literature, the present investigation is anticipated to furnish valuable information for the design of efficient heat transport systems in high-magnetic-field applications.

\section{Numerical Implementation}

\section{A. Computational Set-up}

In the present investigation, a flow of electrically conducting fluid with electric current pulses injected from one of the out-of-plane walls is considered [11] (as depicted in Fig. 1). The bottom wall of the duct is uniformly heated. A constant uniform magnetic field is imposed in the axial direction. A quasi-two-dimensional (quasi-2D) model for MHD duct flow developed by Sommeria and Moreau [12] is employed (hereafter SM82). Generally the SM82 model is applicable for MHD duct flows under the influence of a strong transverse magnetic field, although some deviations 
from quasi-2D behavior can be observed in some situations, e.g. in complex geometry ducts.

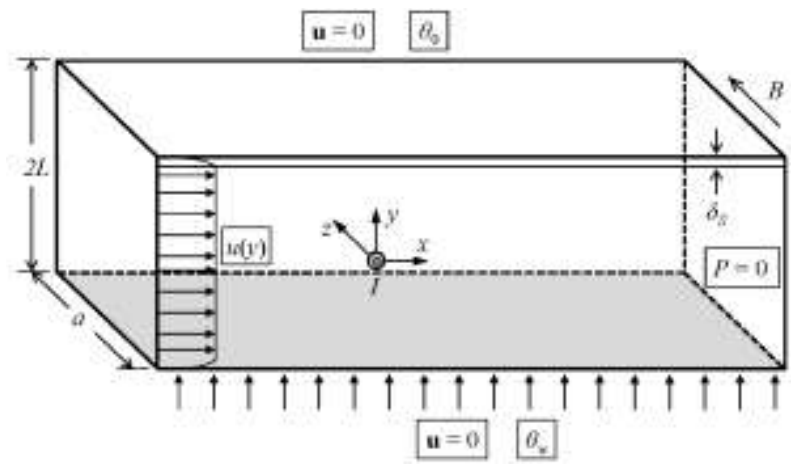

Figure 1. Schematic diagram of the system under investigation. The circle indicates a point electrode mounted in one of the Hartmann wall.

Under the SM82 model the non-dimensional magnetohydrodynamic equations of continuity, momentum and energy reduce to

$$
\nabla \cdot \mathbf{u}=0
$$

$$
\begin{gathered}
\frac{\partial \mathbf{u}}{\partial t}=-(\mathbf{u} \cdot \nabla) \mathbf{u}-\nabla p+\frac{1}{R e_{L}} \nabla 2 \mathbf{u}+\frac{H}{R e_{L}}\left(\frac{1}{n} \mathbf{u}_{0}-\mathbf{u}\right), \\
\frac{\partial \theta}{\partial t}+(\mathbf{u} \cdot \nabla)=\frac{1}{P e} \nabla^{2} \theta
\end{gathered}
$$

respectively. $\mathbf{u}, p$ and $\theta$ are the velocity, pressure and temperature fields, respectively, projected onto a plane orthogonal to the magnetic field, $\nabla$ is the gradient operator and $\mathbf{u}_{0}$ is the force vector field [13]. The dimensionless parameters Reynolds number, Hartmann friction parameter and Peclet number are defined as $R e_{L}=U_{0} L / v, H=(L / a)^{2} n B a \sqrt{\sigma / \rho v}$ and $P e=U_{0} L / k$, where $L$ is half duct width, $U_{0}$ is peak inlet velocity, $B$ is imposed magnetic field, $a$ is out-of-plane duct depth, $n$ is the number of Hartmann walls (in the present study, $n=2$ ), $\sigma, \rho, v$ and $k$ are magnetic permeability, density, kinematic viscosity and thermal conductivity of the liquid metal, respectively. Here length is scaled by $L$, velocity by $U_{0}$, pressure by $\rho U_{0}^{2}$, time by $L / U_{0}$ and temperature by the temperature difference between the bottom and top walls, $\Delta \theta$.

An advanced, high-order, in-house solver based on a spectral element method for spatial discretization is employed to simulate the cases. The implementation of the SM82 model within the solver has previously been validated in $[5,6,13,14]$, and those papers should be consulted for further details.

\section{B. Grid Independence Study}

The optimum fineness of the mesh required for the current problems was evaluated by conducting a grid independence study. The element polynomial degree was varied from 4 to 9, while keeping the macro element distribution unchanged. The grid consists of four regions: two regions near the transverse walls, a region centered at the electrode location and a core region (as shown in Fig. 2).

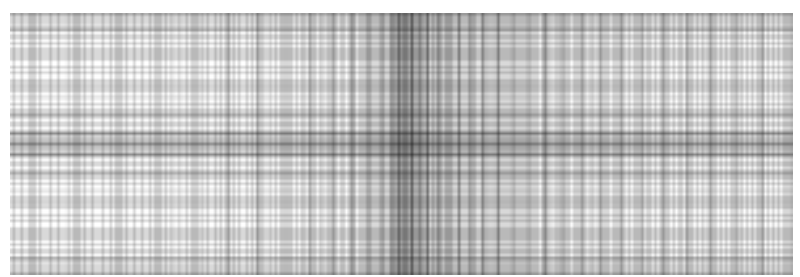

Figure 2. Close-up of the spectrally discretised domain using an $8^{\text {th }}$ order polynomial shape function. The mesh in the vicinity of the electrode is shown $(\approx 20 \%$ of the full-size domain). The full mesh extends $3.2 L$ upstream and $8 L$ downstream.

Convergence is assessed using the time-averaged Strouhal number $(S t)$, total drag coefficient $(C D)$ and Nusselt number $(\mathrm{Nu})$, where the error of these quantities is defined as $\varepsilon_{P}=\left|1-P_{N i} P_{N=11}\right| \times 100 \%$, where $P$ is the monitored parameter. Results, presented in Table 1, reveal that a mesh with $N_{p}=8$ has a numerical convergence that is better than $0.1 \%$, and thus was chosen for the present analysis.

TABLE I. GRID INDEPENDENCE STUDY AT $H=500$, $R e_{L}=1500, I=24, \omega_{f}=5$ AND $\tau / T=0.25$. A POLYNOMIAL DEGREE OF $N_{p}=8$ WAS CHOSEN FOR THE SIMULATIONS IN THE PRESENT STUDY.

\begin{tabular}{|c|c|c|c|}
\hline$N_{p}$ & $\varepsilon_{S t}$ & $\varepsilon_{C D}$ & $\varepsilon_{N u}$ \\
\hline 4 & 0.3813 & 6.6498 & 0.4072 \\
\hline 5 & 0.1259 & 0.4469 & 0.1220 \\
\hline 6 & 0.0495 & 0.1388 & 0.1185 \\
\hline 7 & 0.0193 & 0.4423 & 0.2748 \\
\hline 8 & 0.0193 & 0.0971 & 0.0337 \\
\hline 9 & 0.0443 & 0.2343 & 0.0698 \\
\hline
\end{tabular}

\section{Results}

This section reports on the influence of magnetic field strength (quantified by friction parameter $H$ ), Reynolds number and current injection amplitude on the Nusselt number and the heat transfer enhancement ratio. Other parameters are fixed at $\omega_{f}=1.75$ and $\tau / T=0.25$, where $\omega_{f}$ and $\tau / T$ are the current injection angular frequency and pulse width, respectively.

\section{A. Nusselt Number}

The thermal performance of an MHD duct flow was quantified by the time-averaged Nusselt number, i.e.

$$
N u=1 /\left(t L_{w}\right) \int_{0}^{L_{w}} \int_{0}^{t} N u_{w}(x, t) \mathrm{d} t \mathrm{~d} x,
$$

where $L_{w}$ is the length of the heated bottom wall. The results are presented in Fig. 3 for $200 \leq H \leq 2500$ and $R e_{L}=$ 1500 and 3000. 
Proc. of Fifth International Conference On Advances in Civil, Structural and Mechanical Engineering -ACSM 2016 Copyright @ Institute of Research Engineers and Doctors, USA .All rights reserved.

ISBN: 978-1-63248-105-4 doi: 10.15224/ 978-1-63248-105-4-23

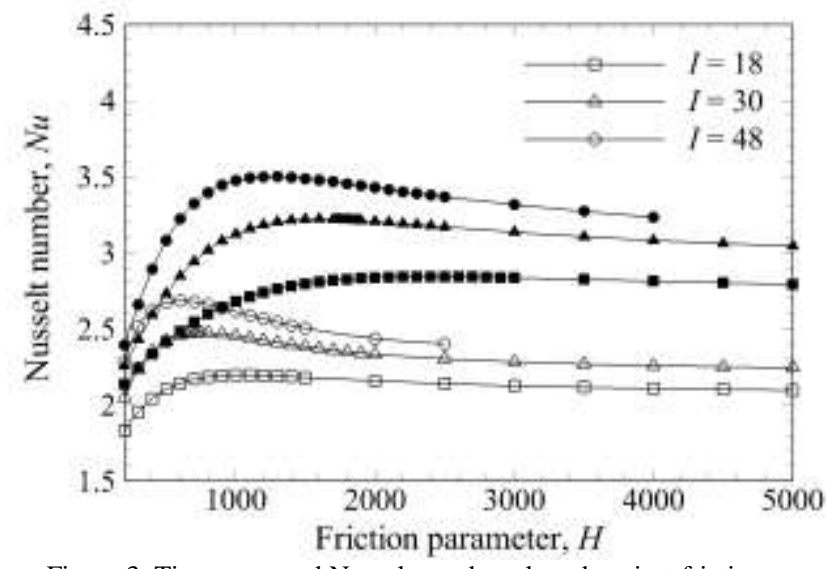

Figure 3. Time-averaged Nusselt number plotted against friction parameter for current amplitude as indicated. Open and solid symbols represent $R e_{L}=1500$ and 3000, respectively.

The figure shows a non-monotonic relation between the Nusselt number and the friction parameter. Hamid, et al. [13] explained that this is due to the competition between the driving Lorentz force and the Hartmann damping. In the low- $H$ regime, an increase in friction parameter yield stronger electrically driven vortices. These vortices advect to a greater downstream distance while interacting with the heated wall boundary layer (as shown by in Fig. 4(a)), thus leading to an increased heat transfer enhancement. There is an almost consistent enhancement in the local Nusselt number along the downstream of the duct (as evidenced in Fig. 5).

(a)

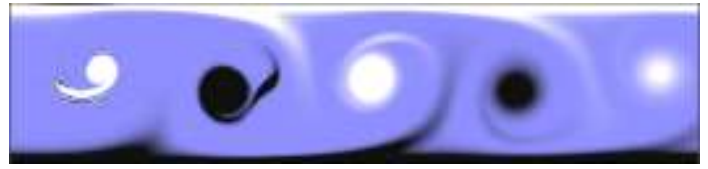

(b)

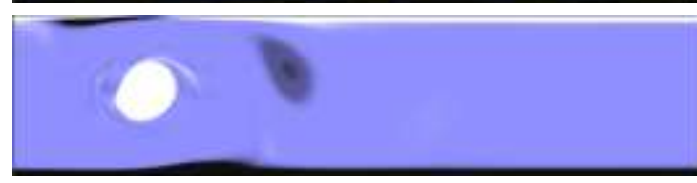

Figure 4. Instantaneous vorticity contour plots for $R e_{L}=1500, I=30$ and (a) $H=400$ and (b) $H=2000$. Contour levels ranges between -2 and 2, with light and dark contours represent positive and negative vorticity, respectively.

However, in the high- $H$ regime, the action of Hartmann damping is more prominent than the driving Lorentz force. This leads to strong vortices only in the vicinity of the injection point, followed by a rapid decay after its formation, as shown in Fig. 4(b). As a result, a substantial enhancement in heat transfer which is localized only in this region was observed, as indicated by a strong peak in the local Nusselt number plot in Fig. 5. The peak is followed by a rapid decline further downstream due to the damping of vortices.

It is also noted from Fig. 3 that higher $R e_{L}$ leads to a better convective heat transfer due to the increased level of turbulence, which is favourable for effective heat dissipation [3]. It is further noted that the $N u$ increases as current injection amplitude increases. This may be attributed to the fact that larger current amplitude induces stronger vortices, which leads to a stronger thermal layer entrainment towards the opposite relatively colder wall and thus better heat transfer. However, there is a diminishing benefit in terms of convective heat transfer as the current amplitude is progressively increased.

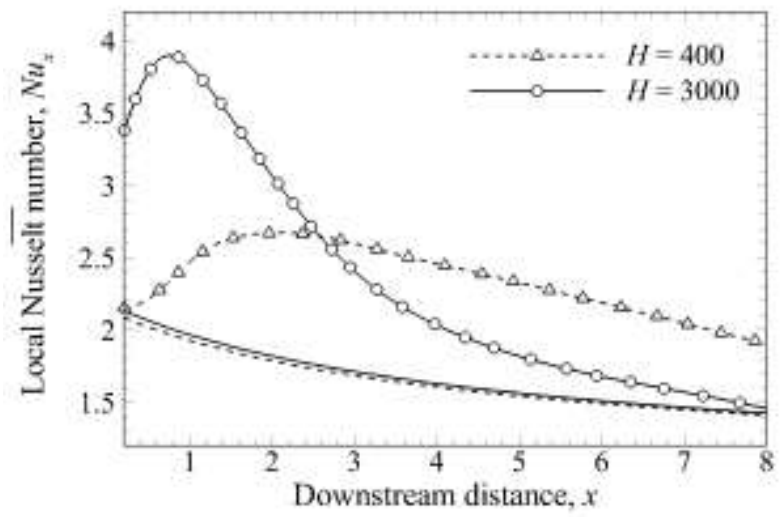

Figure 5. Time-averaged local Nusselt number along the downstream of the heated wall for $R e_{L}=1500, I=30$ and friction parameter as indicated. Lines without symbols represent the corresponding base cases without current injection.

\section{B. Heat Transfer Enhancement Ratio}

The data from Fig. 3 were replotted as a function of the heat transfer enhancement ratio, i.e.

$$
H R=N u / N u_{0},
$$

where $N u_{0}$ is the time-averaged Nusselt number of the heated region of the duct without any current injection, as shown in Fig. 6.

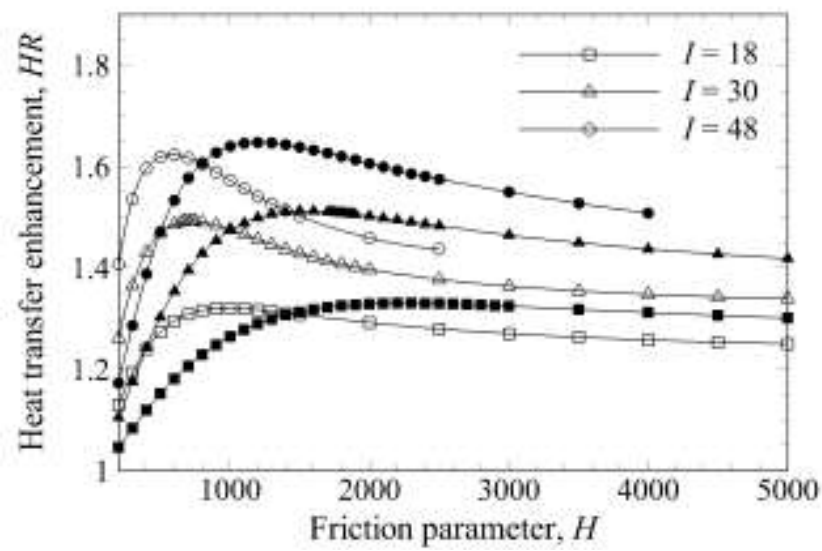

Figure 6. Heat transfer enhancement ratio plotted against friction parameter for current amplitude as indicated. Symbols are as per Fig. 1.

Interestingly, the figure indicates that the maximum attainable heat transfer enhancement is almost uninfluenced by the Reynolds number. This is due to the counterbalancing effect that the Nusselt number for both cases with and without current injection is progressively increases as the Reynolds number increases. It is also observed that for a higher current amplitude, higher Reynolds number flows outperform the lower one for almost any given friction parameter.

The resulting heat transfer enhancement ratio variation is plotted against the ratio of friction parameter to Reynolds number $H / R_{L}$; following Fig. 6 of Sukoriansky, et al. [3], and is presented here in Fig. 7. 


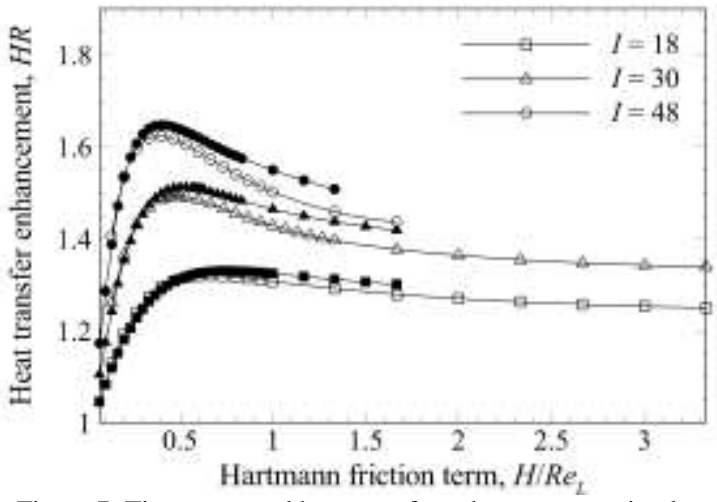

Figure 7. Time-averaged heat transfer enhancement ratio plotted against $H / R e_{L}$ for current amplitude as indicated. Symbols are as per Fig. 1.

It is obvious from this figure that higher current injection amplitude leads to a greater heat transfer enhancement. Furthermore, Fig. 7 also shows that in the low- $H / R e_{L}$ regime (a regime where $H / R e_{L}<\left(H / \operatorname{Re}_{L}\right)_{c r}$, where $\left(H / R e_{L}\right)_{c r}$ is the critical value corresponding to the peak of $H R$ for the respective constant Reynolds number or friction parameter lines), an increase in $H / R e_{L}$ leads to an increase in the heat transfer enhancement ratio. This is due to the enhancement in the local Nusselt number in the region closer to the electrode (as indicated by region $S 2$ in Fig. 8) being greater than its diminution further downstream (as represented by region $D 2$ ), which results in a net increase in $H R$. However, the opposite is true in the high-H/Re $e_{L}$ regime. This is due to the shifting of the intersection point towards the upstream with increasing $H / R e_{L}$ (e.g. from points $I 2$ to $I 1$ as shown in Fig. 8), resulting in a simultaneous shrinking and growing of the "surplus" $S 1$ and "deficit" $D 1$ regions, respectively, and leading to a net decrease in $H R$.

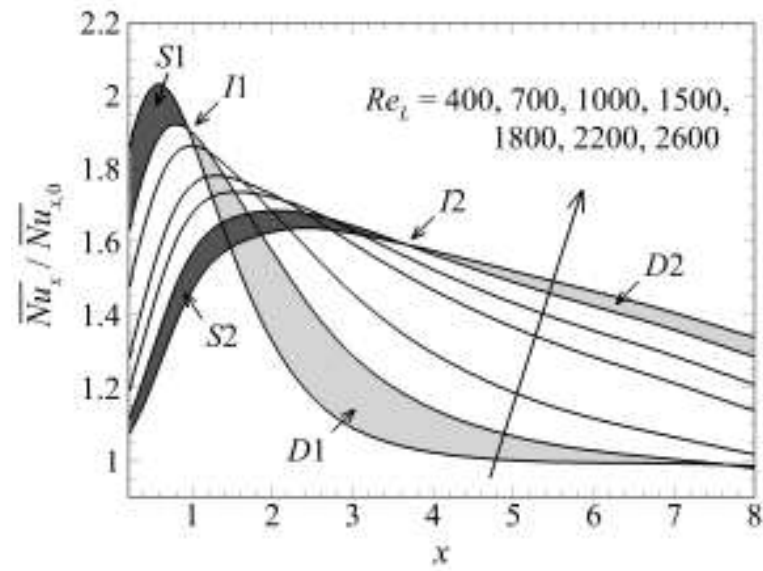

Figure 8. Normalised time-averaged local Nusselt number along the downstream of the heated wall for $H=1000$ and $I=30$. The dark (lighter) regions correspond to a deficit (surplus) in heat transfer enhancement of a higher Reynolds number case relative to the lower one. $I 1$ and $I 2$ are intersection points of local Nusselt nuber lines at different Reynolds number.

It is also interesting to note from Fig. 7 that the $H R$ nearly collapses in the low-H/Re $e_{L}$ regime, but not in the higher range of $H / R e_{L}$. This is despite the fact that, in the limit of high magnetic field strength and for a given $H / R_{L}$, the resultant vortex pattern and strength are similar regardless of both the Reynolds number and the friction parameter (which is expected since the birth of the vortices and their subsequent decay are governed by the ratio of $H / R e_{L}$, as indicated by the respective forcing terms in Eq. (2)). In the high-H/Re $e_{L}$ regime, the magnetic damping effect on the bulk flow is relatively significant (i.e. the heat transport is conductively dominated), thus an increase in Reynolds number yields an appreciable thinning in the thermal boundary layer (as evidenced in Fig. 9) and as a result an increase in the heat transfer enhancement ratio. In the low-H/Re $e_{L}$ regime, however, the flow is already convectively dominated and thus is less sensitive to Reynolds number variations.

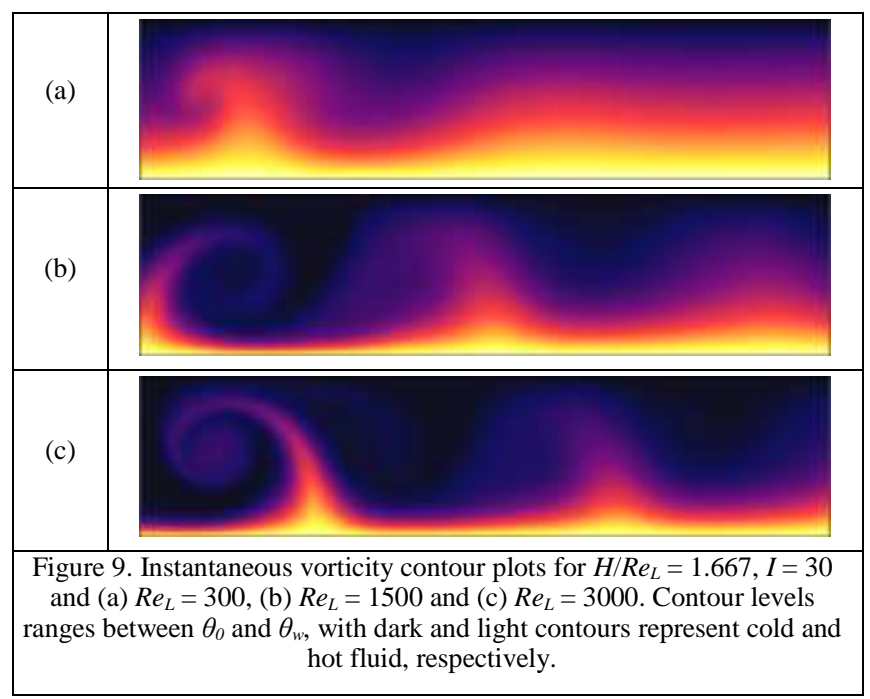

\section{Conclusion}

The present investigation has explored the potential of electrically driven vortices as a mean of enhancing convective heat transfer from a heated wall. The results revealed that the Nusselt number and the heat transfer enhancement ratio have a non-monotonic relation with friction parameter, regardless of Reynolds number and current amplitude. Although the maximum Nusselt number increases with increasing Reynolds number for a given current amplitude, the maximum heat transfer enhancement ratio is almost uninfluenced by the Reynolds number. The enhancement in heat transfer is closely associated with the strength of the vortex-thermal boundary layer interaction.

Further analysis revealed that the heat transfer enhancement is governed by the ratio of friction parameter to Reynolds number, particularly in the low- $H / R e_{L}$ regime. However, in the high- $H / R_{L}$ regime, the heat transfer is conductive. Thus, an increase in Reynolds number results in an increase in the heat transfer enhancement ratio. Given the fact that the electrically generated vortices can be precisely controlled via current injection parameters, current injection vortex promoter stand as a promising technology superior to its physical counterparts, notwithstanding the significant technical challenges in their deployment.

\section{Acknowledgment}

This research was supported by the Australian Research Council through Discovery Grants DP120100153 and 
DP150102920, a high-performance computing time allocations from the National Computational Infrastructure (NCI), which is supported by the Australian Government, and the Monash SunGRID. A. H. A. H. is supported by the Malaysia Ministry of Education and the Universiti Teknologi MARA, Malaysia.

\section{References}

[1] F. Dobran, "Fusion energy conversion in magnetically confined plasma reactors," Prog. Nucl. Energ., vol. 60, pp. 89-116, 2012.

[2] S. Malang and M. Tillack, "Development of self-cooled liquid metal breeder blankets," Forschungszentrum Karlsruhe GmbH, Karlsruhe FZKA 5581, 1995

[3] S. Sukoriansky, D. Klaiman, H. Branover, and E. Greenspan, "MHD enhancement of heat transfer and its relevance to fusion reactor blanket design," Fusion Eng. and Des., vol. 8, pp. 277-282, 1989.

[4] H. Branover, A. Eidelman, and M. Nagorny, "Use of turbulence modification for heat transfer enhancement in liquid metal blankets," Fusion Engineering and Design, vol. 27, pp. 719-724, 1995.

[5] W. K. Hussam and G. J. Sheard, "Heat transfer in a high Hartmann number MHD duct flow with a circular cylinder placed near the heated side-wall," International Journal of Heat and Mass Transfer, vol. 67, pp. 944-954, 2013.

[6] W. K. Hussam, M. C. Thompson, and G. J. Sheard, "Enhancing heat transfer in a high Hartmann number magnetohydrodynamic channel flow via torsional oscillation of a cylindrical obstacle," Physics of Fluids (1994-present), vol. 24, p. 113601, 2012.

[7] L. Bühler, "Instabilities in quasi-two-dimensional magnetohydrodynamic flows," J. Fluid Mech., vol. 326, pp. 125-150, 1996.

[8] S. Kenjereš, "Energy spectra and turbulence generation in the wake of magnetic obstacles," Phys. Fluids, vol. 24, p. 115111, 2012.

[9] C. Reed, B. Picologlou, T. Hua, and J. Walker, "ALEX results-a comparison of measurements from a round and a rectangular duct with 3-D code predictions," in IEEE 12th Symposium on Fusion Engineering, 1987, pp. 1267-1270.

[10] A. Pothérat and R. Klein, "Why, how and when MHD turbulence at low becomes three-dimensional," Journal of Fluid Mechanics, vol. 761, pp. 168-205, 2014.

[11] A. H. A. Hamid, W. K. Hussam, and G. J. Sheard, "Heat transfer augmentation of a quasi-two-dimensional MHD duct flow via electrically driven vortices," Numer. Heat Tr. A-Appl., In Press.

[12] J. Sommeria and R. Moreau, "Why, how, and when, MHD turbulence becomes two-dimensional," J. Fluid Mech., vol. 118, pp. 507-518, 1982

[13] A. H. A. Hamid, W. K. Hussam, and G. J. Sheard, "Combining an obstacle and electrically driven vortices to enhance heat transfer in a quasi-two-dimensional MHD duct flow," J. Fluid Mech., vol. 792, pp. 364-396, 2016

[14] A. H. A. Hamid, W. K. Hussam, A. Pothérat, and G. J. Sheard, "Spatial evolution of a quasi-two-dimensional Kármán vortex street subjected to a strong uniform magnetic field," Phys. Fluids, vol. 27, p. $053602,2015$.
About Author (s)

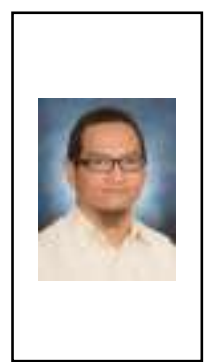

Ahmad H. A. Hamid is an academic staff in the Faculty of Mechanical Engineering, Universiti Teknologi MARA, Malaysia.

A. H. A. Hamid received his M.Sc. degree in 2008 from the same university. His research interests include wake flow of a bluff body, magnetohydrodynamic flow and heat transfer.

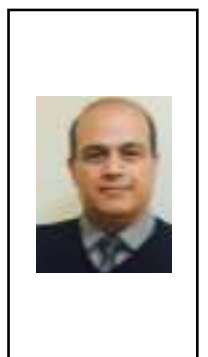

Wisam K. Hussam is a Research Associate at the Faculty of Mechanical Engineering, Monash University Australia. He completed his MSc from Al-Mustansiriya University, Iraq in 2001, and his PhD from Monash University, Australia in 2012. His research interests focus on heat transfer enhancement, instability and transition in fluid flow, natural convection for Geophysical flow and Nanofluids.

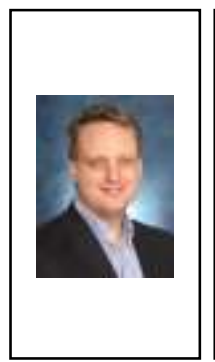

A/Prof. Gregory J. Sheard is a Faculty member of the Department of Mechanical and Aerospace Engineering, Monash University, Australia. Since 2001 he has published 120 papers on the simulation of fluid flows and their stability, convection and heat transfer, wakes, rotating flows and magnetohydrodynamics. 